\title{
Interactive comment on "Potential-Vorticity
}

Dynamics of Troughs and Ridges within Rossby

Wave Packets during a 40-year reanalysis period" by Franziska Teubler and Michael Riemer

\section{Franziska Teubler and Michael Riemer}

f.teubler@uni-mainz.de

Received and published: 23 December 2020

The comment was uploaded in the form of a supplement:

https://wcd.copernicus.org/preprints/wcd-2020-52/wcd-2020-52-AC1-supplement.pdf

Interactive comment on Weather Clim. Dynam. Discuss., https://doi.org/10.5194/wcd-2020-52, 2020. 